\title{
Immediate intraocular pressure response to selective laser trabeculoplasty
}

\author{
Paolo Lanzetta, Ugo Menchini, Gianni Virgili
}

\begin{abstract}
Backgroundlaims-Selective

laser trabeculoplasty targets the pigmented trabecular meshwork cells without damage to the trabecular meshwork architecture in vitro. A study was conducted in vivo of eight eyes with uncontrolled open angle glaucoma to ascertain the immediate intraocular response to selective laser trabeculoplasty.

Methods-The trabecular meshwork of each eye was treated $360^{\circ}$ with a frequency doubled Q-switched Nd:YAG laser. Intraocular pressure was measured 1, 2, 24 hours and 1, 4, 6 weeks after treatment. Results-The average preoperative intraocular pressure was 26.6 (SD 7) $\mathrm{mm} \mathrm{Hg}$ (range 18-37). Two hours and 6 weeks respectively after selective trabeculoplasty intraocular pressure was reduced in all the eyes treated with an average fall of $\mathbf{1 0 . 6}$ (5.2) $\mathrm{mm} \mathrm{Hg}$ or $39.9 \%$. A pressure spike of $10 \mathrm{~mm} \mathrm{Hg}$ verified in one eye 1 hour after treatment.

Conclusions-Selective laser trabeculoplasty decreased intraocular pressure by an amount similar to that achieved with standard trabeculoplasty. Additional study is needed to determine whether the beneficial effect is sustained over a longer period of follow up.

(Br f Ophthalmol 1999;83:29-32)
\end{abstract}

Argon laser trabeculoplasty (ALT) is a well established therapy for lowering intraocular pressure (IOP) in eyes with chronic open angle glaucoma. ${ }^{1}$ Whether the exact mechanism of action of ALT is purely mechanical, ${ }^{23}$ cellular, ${ }^{4-6}$ or a combination of both it is widely accepted that ALT causes an enhancement of egress of fluid via the trabecular outflow system resulting in lowered IOP. However, histopathological examination of laser treated meshwork samples obtained from human subjects revealed early necrosis of cells and disruption of trabecular beams. A shrinkage of certain collagenous elements was also associated to treatment. ${ }^{6-11}$ Thus, ALT is fundamentally destructive to trabecular meshwork as it focally destroys and alters the biological activity of trabecular cells temporarily ameliorating IOP. ALT uses continuous wave lasers with pulse duration in the millisecond range or greater. With such pulse duration heat is transmitted from pigmented targeted cells to adjacent tissues and subsequent damage of non-pigmented cells and trabecular beams occurs.
Recently Latina and Park $^{12}$ used a $532 \mathrm{~nm}$ Q-switched Nd:YAG laser to selectively target pigmented trabecular meshwork cells in vitro with low threshold radiant exposures without producing collateral damage to adjacent nonpigmented cells or structures. This laser gives a single pulse of short duration (in the microsecond range) and low fluence (energy/area). At these values, unlike in ALT, thermal diffusion from targeted cells to surrounding nonpigmented trabecular meshwork cells is minimised; therefore, the technique has been named selective laser trabeculoplasty (SLT). A histological study in human cadaver eyes treated with SLT or ALT within 18 hours of death confirmed that selective treatment appears to cause no coagulation damage to the trabecular meshwork and less structural damage than ALT. ${ }^{13}$

Following the results of the study by Latina and Park $^{12}$ the clinical application of a Q-switched Nd:YAG laser for SLT was proposed as a new laser treatment for chronic open angle glaucoma. ${ }^{14}$ The possibility of avoiding relevant thermal or coagulative damage in the trabecular meshwork with SLT represents a major improvement over conventional ALT. As a consequence SLT might be applied many times in the same eye without determining coagulative scarring that focally reduces or prevents aqueous outflow. This study presents preliminary results on the immediate IOP response to SLT in eight eyes with uncontrolled open angle glaucoma.

Subjects and methods

Eight eyes of six patients (four male, two female) with primary open angle glaucoma were treated with SLT. The patients ranged in age from 59 to 84 years (mean 72.3 (SD 11.2)). All the eyes were undergoing the maximum tolerated medical therapy (three eyes $\beta$ blockers and pilocarpine; two eyes $\beta$ blockers and topical carbonic anhydrase inhibitors (CAIs); one eye $\beta$ blockers, pilocarpine, and topical CAIs; one eye $\beta$ blockers, topical CAIs, and latanoprost; one eye $\beta$ blockers, pilocarpine, topical CAIs, and apraclonidine). Three eyes had previous ALT and one eye received a trabeculectomy 1 year before SLT. All the patients had been fully informed about the procedure and informed consent was obtained from all of them.

The grade of pigmentation of the trabecular meshwork was registered before treatment. In one eye the trabecular meshwork appeared grey in colour with a trace of pigmentation while no pigmentation was detected on
Accepted for publication 26 August 1998 
Table 1 Results of selective laser trabeculoplasty

\begin{tabular}{|c|c|c|c|c|c|c|c|c|}
\hline \multirow[b]{2}{*}{ Case; patient } & \multirow[b]{2}{*}{ Preop Tx } & \multirow[b]{2}{*}{ Preop IOP } & \multicolumn{6}{|l|}{ IOP; variation } \\
\hline & & & 1 hour postop & 2 hours postop & 24 hours postop & 7 days postop & 4 weeks postop & 6 weeks postop \\
\hline $1 ; 1$ & $\operatorname{Max} T x$, ALT & 18 & $12 ;-6$ & $12 ;-6$ & $10 ;-8$ & $13 ;-5$ & $11 ;-7$ & $13 ;-5$ \\
\hline $2 ; 2$ & $\operatorname{Max} \mathrm{Tx}$ & 31 & $24 ;-7$ & $16 ;-15$ & $16 ;-15$ & $26 ;-5$ & $22 ;-9$ & $20 ;-11$ \\
\hline $3 ; 3$ & $\operatorname{Max} T x$ & 30 & $40 ;+10$ & $28 ;-2$ & $26 ;-4$ & $20 ;-10$ & $20 ;-10$ & $19 ;-11$ \\
\hline $4 ; 4$ & Max Tx, ALT, Trab & 24 & $19 ;-5$ & $16 ;-8$ & $16 ;-8$ & $16 ;-8$ & $13 ;-11$ & $14 ;-10$ \\
\hline $5 ; 5$ & Max Tx, ALT & 20 & $19 ;-1$ & $15 ;-5$ & $11 ;-9$ & $14 ;-6$ & $14 ;-6$ & $14 ;-6$ \\
\hline $6 ; 5$ & $\operatorname{Max} T x$ & 20 & $14 ;-6$ & $13 ;-7$ & $14 ;-6$ & $14 ;-6$ & $14 ;-6$ & $14 ;-6$ \\
\hline $7 ; 6$ & $\operatorname{Max} \mathrm{Tx}$ & 33 & $11 ;-22$ & $12 ;-21$ & $18 ;-15$ & $18 ;-15$ & $18 ;-15$ & $17 ;-16$ \\
\hline \multirow[t]{3}{*}{$8 ; 6$} & $\operatorname{Max} T x$ & 37 & $32 ;-5$ & $16 ;-21$ & $16 ;-21$ & $16 ;-21$ & $16 ;-21$ & $17 ;-20$ \\
\hline & Mean & 26.6 & $21.4 ;-7.8$ & $16 ;-10.6$ & $15.9 ;-10.8$ & $17.1 ;-9.5$ & $16 ;-10.6$ & $16 ;-10.6$ \\
\hline & $\mathrm{SD}$ & 7 & $10.2 ; 6.3$ & $5.2 ; 7.4$ & $4.9 ; 5.7$ & $4.3 ; 5.7$ & $3.7 ; 5.2$ & $2.6 ; 5.2$ \\
\hline
\end{tabular}

$\mathrm{Tx}=$ therapy Max $\mathrm{Tx}=$ maximal medical therapy; $\mathrm{ALT}=$ argon laser trabeculoplasty; $\mathrm{Trab}=$ trabeculectomy.

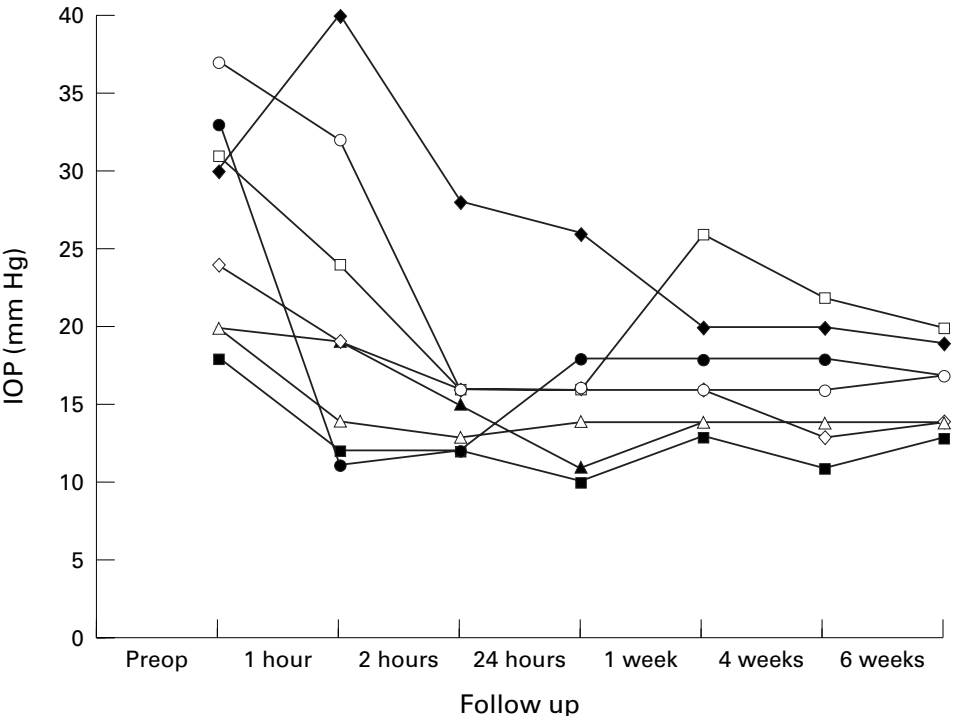

Figure 1 Distribution of IOP before and after selective laser trabeculoplasty.

Schwalbe's line. In the remaining eyes the trabecular meshwork appeared light to medium brown in colour; pigmentation was mostly confined to the trabecular meshwork and sparse pigmentation was visible on Schwalbe's line.

Following topical anaesthesia with oxybuprocaine $0.4 \%$ eye drops a three mirror Goldmann lens was applied. The trabecular meshwork of each eye was treated $360^{\circ}$ with a 532 nm frequency doubled Q-switched Nd:YAG laser (Selecta 7000; Coherent, Palo Alto, CA, USA). The pulse duration was 3 ns with a single pulse. The spot size was $400 \mu \mathrm{m}$. Energies were between $0.70 \mathrm{~mJ}$ and $1.10 \mathrm{~mJ}$ with a mean energy of $0.82 \mathrm{~mJ}$ for each spot. Mean total energy delivered was $39.27 \mathrm{~mJ}$ (range $32.9-47.2 \mathrm{~mJ}$ ). The mean number of spots applied was 49.1 (range 40-60). Treatment was conducted with adjacent but not overlapping spots. If bubble formation was observed energy was decreased by $0.1 \mathrm{~mJ}$ increments until bubbling disappeared. Indomethacin $0.1 \%$ eye drops were prescribed after treatment three times a day for 10 days. Hypotensive medical therapy was not modified after treatment. All the eyes underwent a slit lamp examination and applanation tonometry 1 hour, 2 hours, 24 hours, 7 days, 4 weeks, and 6 weeks after treatment. All patients completed a follow up of 6 weeks.
Results

The average preoperative IOP was 26.6 (SD 7) $\mathrm{mm} \mathrm{Hg}$. Preoperative and postoperative IOP and IOP variation for each eye are shown in Table 1. One hour after treatment seven eyes showed a fall in IOP ranging from 1 to $22 \mathrm{~mm}$ $\mathrm{Hg}$. In one eye a postoperative pressure spike of $10 \mathrm{~mm} \mathrm{Hg}$ was registered and dissipated quickly. At the following examinations all the eyes treated had a reduction of IOP with a fall ranging from $2-21 \mathrm{~mm} \mathrm{Hg}$ by 2 hours, 4-21 $\mathrm{mm} \mathrm{Hg}$ by 24 hours, 5-21 mm Hg by 7 days, 6-21 $\mathrm{mm} \mathrm{Hg}$ by 4 weeks, and $5-20 \mathrm{~mm} \mathrm{Hg}$ by 6 weeks. One and 2 hours after SLT the mean IOP level was 21.4 (SD 10.2) $\mathrm{mm} \mathrm{Hg}$ (range 11-40) and 16 (5.2) $\mathrm{mm} \mathrm{Hg} \mathrm{(12-28)} \mathrm{respec-}$ tively. Twenty four hours after surgery the mean IOP level was 15.9 (4.9) $\mathrm{mm} \mathrm{Hg}(10-26)$. The mean IOP stabilised at 17.1 (4.3) $\mathrm{mm} \mathrm{Hg}$ (1326) by 7 days, 16 (3.7) $\mathrm{mm} \mathrm{Hg}(11-22)$ by 4 weeks, and 16 (2.6) $\mathrm{mm} \mathrm{Hg}(13-20)$ by 6 weeks. Therefore, the mean IOP was reduced by 10.6 (5.2) $\mathrm{mm} \mathrm{Hg}$ or $39.9 \% 6$ weeks after surgery. Figure 1 shows the distribution of IOP values before and after treatment. Significant anterior segment inflammation was not detected after SLT in any of the eyes. None of the patients complained of pain or any discomfort during treatment.

\section{Discussion}

In 1979 Wise and Witter ${ }^{15}$ demonstrated that ALT was successful in reducing IOP during open angle glaucoma. In a pilot study the trabecular meshwork was treated $360^{\circ}$ with 100 evenly spaced burns of $50 \mu \mathrm{m}$ spot size with a power of $1000-1500 \mathrm{~mW}$ for 0.1 second. The average fall in IOP was $10.3 \mathrm{~mm}$ $\mathrm{Hg}$ at 3 months in 41 patients. The current technique for ALT is substantially the same and significantly reduces IOP in the short and medium term. Although the power and duration settings were substantially different from those currently applied, Worthen and Wickham ${ }^{16}$ in 1974 obtained an average IOP reduction of $9.6(8.9) \mathrm{mm} \mathrm{Hg}$ in 20 patients 3 weeks after the treatment. In a group of patients with primary open angle glaucoma on maximum medical therapy, Thomas et al ${ }^{17}$ registered a reduction of $6.4 \mathrm{~mm} \mathrm{Hg}(26 \%)$ at 5 months after ALT. A greater reduction with a fall of $9.0 \mathrm{~mm} \mathrm{Hg}(33 \%)$ at 3 months is usually obtained when ALT is used as a primary treatment. $^{18}$ In general, pressure reduction 
with ALT is seldom greater than $30 \%$ and the time course of the ALT response is relatively slow. ${ }^{4}$

Alexander and Grierson ${ }^{19}$ showed that the ultrastructural changes of the human trabecular meshwork after ALT comprise both destructive and stimulating events. The primary effect of laser irradiation is related to a thermal injury induced at the melanin containing trabecular meshwork cells, which represent the major absorption site of laser wavelength. Concurrent damage to adjacent nonpigmented cells and collagen trabecular beams occurs as a result of heat transmission. ALT induces intratrabecular debris, distortion of trabecular beams, and death of trabecular endothelial cells, leaving the damaged beams denuded. There is also evidence of activation and migration of surviving trabecular endothelial cells to the burn site. ${ }^{4}$ Destructive mechanisms and excessive repair response such as cellular sheets extending from Schwalbe's line and covering the surface of the anterior meshwork are related to failure of the laser treatment. Conversely, laser stimulated trabecular cell division after ALT may play a positive influence on outflow resistance by causing alterations in the synthesis of the proteoglycan components of the extracellular matrix. ${ }^{20}$ Although the biological mechanism of action of ALT is still poorly understood this histological evidence suggests that irradiation to the trabecular meshwork stimulates therapeutic benefits and that the damage to the structural integrity of the trabecular meshwork is redundant and should be avoided. Therefore a selective low threshold targeting of pigmented trabecular meshwork cells, without collateral thermal damage to the adjacent nonpigmented trabecular meshwork cells and underlying trabecular beams should become the desired endpoint of a therapeutically effective laser trabeculoplasty. At irradiances below threshold no damage to either pigmented or non-pigmented trabecular meshwork cells occurs; at threshold irradiances energy is transmitted from pigmented to non-pigmented cells resulting in a non-selective targeting. At irradiances higher than threshold trabecular meshwork cells are completely vaporised and irreversibly damaged. ${ }^{12}$ The visible endpoint during ALT corresponds to overthreshold irradiation (that is, whitening and bubble formation) resulting in a coagulative necrosis of the trabecular meshwork with non-specific targeting of cells, diffusion of thermal energy to collagen beams, and damage to the Schlemm's canal. ${ }^{21-23}$

In 1995 Latina and Park ${ }^{12}$ introduced a procedure to selectively target pigmented trabecular meshwork cells while sparing adjacent cells and tissues from collateral thermal damage and to preserve the structural integrity of the trabecular meshwork. The authors postulated that a selective treatment could induce an effective clinical response. Selective targeting of pigmented trabecular meshwork cells without adjacent damage to non-pigmented cells and collagen beams can be obtained with pulse duration of $1 \mu$ s or less. Roider et al ${ }^{24}$ showed that for exposure in the range of microseconds the damage may be limited to subcellular molecular structures not detectable by electron microscopy. Latina and Park ${ }^{12}$ demonstrated that a $532 \mathrm{~nm}$ Q-switched Nd:YAG laser with a single pulse of short duration and low energy can be used to selectively target trabecular cells in vitro. Noecker et $a l^{14}$ recently compared the acute morphological changes after SLT and ALT by electron microscopic evaluation of human cadaver eyes. ALT induced crater formation in the uveal meshwork at the junction of the pigmented and non-pigmented trabecular meshwork; coagulative damage was evident at the base and along the edge of craters with whitening bleb formation, disruption of the collagen beams and endothelial cells. In the eyes treated with SLT there was no evidence of coagulative damage and minimal evidence of mechanical damage shown by rare, focal cracks on the trabecular beams. For increasing energy levels, morphological alterations of the trabecular beams and endothelial cells were more prominent. Following these results SLT appears to cause no coagulative damage to the human trabecular meshwork and less damage than ALT.

In our study all the patients were affected with uncontrolled open angle glaucoma on maximum therapy. Three of eight eyes had already received ALT and one had undergone a trabeculectomy. By 6 weeks after SLT an average IOP reduction of $10.6 \mathrm{~mm} \mathrm{Hg}$ $(39.9 \%)$ was obtained. SLT achieved an immediate effect. Within 2 hours after treatment the study patients had a mean IOP decrease of approximately $40 \%$ which maintained after 24 hours. Therefore, it seems that the therapeutic effect of SLT is seen immediately after treatment whereas with ALT the effect may appear at about 6 weeks. ${ }^{25}$ While ALT usually causes a temporary disruption of the blood-ocular barrier, ${ }^{26}$ no evidence of aqueous protein flare was registered within 24 hours after laser irradiation. The most serious complication of ALT is sudden elevation of IOP after treatment. In the Glaucoma Laser Trial Research Group ${ }^{27} 34 \%$ of patients treated with ALT had a IOP rise of $5 \mathrm{~mm} \mathrm{Hg}$ or greater above baseline and $12 \%$ of eyes demonstrated and increase of $10 \mathrm{~mm} \mathrm{Hg}$ or more. An association between post-ALT IOP elevation and laser energy has been postulated. ${ }^{28}$ Although exposure dose studies show that selective targeting can be maintained over an exposure range of 20 times threshold for the wavelength considered ${ }^{12}$ the transient spike in pressure that we observed was associated with higher energies of between 0.8 and $1.10 \mathrm{~mJ}$ (average energy $0.94 \mathrm{~mJ}$ ).

In conclusion, the aim of SLT is to limit the lesion at the pigmented trabecular meshwork cells while sparing non-pigmented cells and collagen trabecular beams. Recent observations in vitro and in cadaver human eyes ${ }^{12} 13$ have shown that pigmented trabecular meshwork cells can be selectively targeted avoiding gross disruption of cellular architecture and without extended and redundant damage to adjacent non-pigmented cells and trabecular 
meshwork architecture. Our preliminary clinical study seems to indicate that SLT with subvisible thermal damage could be therapeutically beneficial in reducing IOP in the immediate postoperative period.

1 Coakes R. Laser trabeculoplasty. Br f Ophthalmol 1992; 76:624-6.

2 Wise JB. Glaucoma treatment by trabecular tightening with the argon laser. Int Ophthalmol Clin 1981;21:69-78.

3 Wise JB. Management of the glaucomas with argon laser (laser trabeculoplasty). In: Boyd FG, ed. Highlights of ophthalmology. Panama: Highlights Press, 1985.

4 Bylsma SB, Samples JR, Acott TS, et al. Trabecular cell division after argon laser trabeculoplasty. Arch Ophthalmol 1988;106:544-7.

5 Gilbert CM, Brown RH, Lynch MG. The effect of argon laser trabeculoplasty on the rate of filtering surgery. Ophthalmology 1986:93:362-5.

6 Van Buskirk EM, Pond V, Resenquist RC, et al Argon laser trabeculoplasty. Studies of mechanism of action. Ophthalmology 1984;91:1005-10.

7 Rodrigues MM, Spaeth GL, Donohoo P. Electron microscopy of argon laser therapy in phakic open-angle glaucoma.

8 Starita RJ, Rodrigues MM, Fellman RL, et al. Histopathologic verification of position of laser burns in argon laser trabeculoplasty. Ophthalmic Surg 1984;15:854-8.

9 Ticho U, Zauberman H. Argon laser application to the angle structures in the glaucomas. Arch Ophthalmol 1976;94:614.

10 Van Buskirk EM. Pathophysiology of laser trabeculoplasty. Surv Ophthalmol 1989;33:264-72.

11 Wickam MG, Worthen DM, Binder PS. Effects of laser trabeculotomy in rhesus monkey rats. Invest Ophthalmol Vis Sci 1977;16:624-8.

12 Latina MA, Park C. Selective targeting of trabecular meshwork cells: in vitro studies of pulsed and CW laser interactions. Exp Eye Res 1995; 60:359-72.

13 Latina MA, Sibayan S. Selective laser trabeculoplasty: a new treatment for glaucoma. In: Laser technology in ophthalmology. Proc Fifth International Congress, Lugano, Switzerland, June 26-29, 1996. Amsterdam: Kugler, 1996:24
14 Noecker RJ, Kramer TR, Latina M, et al. Comparison of acute morphologic changes after selective laser trabeculocute mologic changes after selective laser trabeculoplasty and argon laser trabeculoplasty by electron microSci 1998:39:S472. Abstract no 2161.

15 Wise JB, Witter SL. Argon laser therapy for open-angle glaucoma: a pilot study. Arch Ophthalmol 1979;97:319-22.

6 Worthen DM, Wickam MG. Argon laser trabeculotomy. Trans Am Acad Ophthalmol Otolaryngol 1974;78:371-5.

17 Thomas JV, Simmons RJ, Belcher CD. Argon laser trabecuoplasty in the presurgical glaucoma patient. Ophthalmology 1982;89:187-97.

18 Glaucoma Laser Trial Research Group. The Glaucoma Laser Trial (GLT). II. Results of argon laser trabeculoplasty versus topical medications. Ophthalmology 1990;97: 1403-13.

19 Alexander RA, Grierson I. Morphological effects of argon laser trabeculoplasty upon the glaucomatous human meshwork. Eye 1989;3:719-26.

20 Accott TS, Kingley PD, Samples JR, et al. Human trabecular meshwork organ culture: morphology and glycosaminoglycan

21 van der Zypen E, Fankhauser F. Ultrastructural changes of the trabecular meshwork of the monkey (Macaca speciosa) following irradiation with argon light. Graefes Arch Exp Ophthalmol 1984;221:249-61.

22 Melamed S, Pei J, Epstein DL. Short-term effect of argon aser trabeculoplasty in monkeys. Arch Ophthalmol 1985; 103: $1546-52$.

23 Melamed S, Epstein DL. Alterations of aqueous humor outflow following argon laser trabeculoplasty in monkeys. $\mathrm{Brf}$ Ophthalmol 1987;71:776-81.

24 Roider J, Hillenkamp F, Flotte $\mathrm{T}$, et al. Microphotocoagulation: selective effects of repetitive short laser pulses. Proc Natl Acad Sci USA 1993;90:8643-7.

25 Reiss GR, Wilensky JT, Higginbotham EJ. Laser trabeculoplasty. Surv Ophthalmol 1991;35:407-28.

26 Mermoud A, Pittet N, Herbort CP. Inflammation patterns after laser trabeculoplasty measured with the laser flare meter. Arch Ophthalmol 1992;110:368-70.

27 Glaucoma Laser Trial Research Group. The Glaucoma Laser Trial (GLT). I. Acute effects of argon laser trabeculoplasty on intraocular pressure. Arch Ophthalmol 1984; 107:1135-42.

28 Rosenblatt MA, Luntz MH. Intraocular pressure rise after argon laser trabeculoplasty. Br f Ophthalmol 1987;71:7725 . 